OPEN ACCESS

Edited by:

Stefan de Folter,

Center for Research and Advanced

Studies (CINVESTAV), Mexico

Reviewed by:

Xian Sheng Zhang,

Shandong Agricultural University,

China

Beth Thompson,

East Carolina University, United States

*Correspondence:

Jozef Mravec

mravec@plen.ku.dk

Specialty section:

This article was submitted to

Plant Development and EvoDevo,

a section of the journal

Frontiers in Plant Science

Received: 21 July 2020

Accepted: 31 August 2020

Published: 11 September 2020

Citation:

Lund $\mathrm{CH}$, Stenbæk A, Atmodjo MA, Rasmussen RE, Moller IE, Erstad SM,

Biswal AK, Mohnen D, Mravec J and

Sakuragi Y (2020) Pectin Synthesis and Pollen Tube Growth in

Arabidopsis Involves Three GAUT1

Golgi-Anchoring Proteins: GAUT5,

GAUT6, and GAUT7.

Front. Plant Sci. 11:585774.

doi: 10.3389/fpls.2020.585774

\section{Pectin Synthesis and Pollen Tube Growth in Arabidopsis Involves Three GAUT1 Golgi-Anchoring Proteins: GAUT5, GAUT6, and GAUT7}

\author{
Christian Have Lund ${ }^{1}$, Anne Stenbæk ${ }^{1}$, Melani A. Atmodjo ${ }^{2}$, \\ Randi Engelberth Rasmussen ${ }^{1}$, Isabel E. Moller ${ }^{1}$, Simon Matthé Erstad ${ }^{1}$, \\ Ajaya Kumar Biswal ${ }^{2}$, Debra Mohnen ${ }^{2}$, Jozef Mravec ${ }^{1 *}$ and Yumiko Sakuragi ${ }^{1}$ \\ ${ }^{1}$ Department of Plant and Environmental Sciences, Faculty of Science, University of Copenhagen, Frederiksberg, Denmark, \\ ${ }^{2}$ Department of Biochemistry and Molecular Biology and Complex Carbohydrate Research Center, University of Georgia, \\ Athens, GA, United States
}

The major cell wall pectic glycan homogalacturonan $(\mathrm{HG})$ is crucial for plant growth, development, and reproduction. HG synthesis occurs in the Golgi and is catalyzed by members of the galacturonosyltransferase (GAUT) family with GAUT1 being the archetypal and best studied family member. In Arabidopsis suspension culture cells and tobacco leaves, the Golgi localization of Arabidopsis GAUT1 has been shown to require protein-protein interactions with its homolog GAUT7. Here we show that in pollen tubes GAUT5 and GAUT6, homologs of GAUT7, also target GAUT1 to the Golgi apparatus. Pollen tube germination and elongation in double homozygous knock-out mutants (gaut5 gaut6, gaut5 gaut7, and gaut6 gaut7) are moderately impaired, whereas gaut5 $5^{-/}$gaut6 ${ }^{-/}$gaut $7^{+/-}$triple mutant is severely impaired and male infertile. Amounts and distributions of methylesterified HG in the pollen tube tip were severely distorted in the double and heterozygous triple mutants. A chimeric protein comprising GAUT1 and a non-cleavable membrane anchor domain was able to partially restore pollen tube germination and elongation and to reverse male sterility in the triple mutant. These results indicate that GAUT5, GAUT6, and GAUT7 are required for synthesis of native $\mathrm{HG}$ in growing pollen tubes and have critical roles in pollen tube growth and male fertility in Arabidopsis.

Keywords: cell wall, homogalacturonan, galacturonosyltransferase, pollen, male gametophyte, Golgi apparatus

\section{INTRODUCTION}

Flowering plants (angiosperms) support a myriad of species on earth and have dominated the terrestrial ecosystem since their diversification over the last 130 million years. One of the biological innovations that occurred in angiosperms was rapid pollen tube germination and elongation, which dramatically shortened the reproductive cycle. Pollen tube growth is restricted to the tip region and involves massive secretion and assembly of newly synthesized cell wall materials, of which HG is a major component (Taylor and Hepler, 1997). The pollen tube apical wall is almost exclusively 
composed of a pectin network, while the typical load-bearing cellulose-hemicellulose network is only found in the subapical and more distal parts of the tube (Ferguson et al., 1998). HG is a linear homopolymer of $\alpha$-1,4-linked GalA and is highly esterified during its synthesis in the Golgi apparatus, including methylesterification at the C6 position and, to a lesser extent, acetylesterification at the $\mathrm{O} 2$ and $\mathrm{O} 3$ positions of the GalA residue (Perrone et al., 2002; Pauly and Ramírez, 2018). Highly esterified HG is transported in secretory vesicles and deposited at the tip of growing pollen tubes. HG provides both the elasticity necessary for rapid turgor pressure-driven cell expansion and the rigidity required to withstand the turgor (Dardelle et al., 2010; Chebli et al., 2012).

In the Golgi apparatus, the HG backbone is synthesized in the cis- and medial-cisternae by HG:galacturonosyltransferase (HG : GalAT) and is methyl- and acetyl-esterified by pectin methyltransferases and pectin acetyltransferases (Goubet and Mohnen 1999; Sterling et al., 2001; Caffall and Mohnen, 2009; Atmodjo et al., 2013; Anderson, 2016). Arabidopsis thaliana GALACTURONOSYLTRANSFERASE 1 (GAUT1) was the first identified and biochemically characterized HG : GalAT (Sterling et al., 2006). It belongs to the glycosyltransferase family 8 (GT8) of the CAZy Carbohydrate Active Enzyme database (http://www.cazy. org/; Cantarel et al., 2009) and forms the GAUT gene family together with 14 homologs in Arabidopsis (Sterling et al., 2006; Yin et al., 2010a; Yin et al., 2010b). GAUT1 is a protein of 673 amino acids (aa) with canonical type II transmembrane protein topology which includes a short N-terminal cytosolic tail, a single transmembrane anchor domain, a structurally undefined linker region often referred to as the stem region, and a catalytic domain facing the Golgi lumen (Sterling et al., 2006). Biochemical analyses of GAUT1 in Arabidopsis suspension cultured cells revealed that GAUT1 interacts with GAUT7 (AT2G38650), a homolog with $36 \%$ aa sequence identity to GAUT1, to form a GAUT1:GAUT7 HG : GalAT complex (Atmodjo et al., 2011). Curiously, the catalytic domain of GAUT1 is cleaved in vivo at amino acid position 167 resulting in loss of the single transmembrane domain which generally keeps type II transmembrane proteins in the Golgi. Transient expression studies using fluorescent proteins fused to GAUT1 and GAUT7 in Nicotiana benthamiana leaves revealed that GAUT1 remains tethered to the Golgi membrane through protein-protein interactions with GAUT7 (Atmodjo et al., 2011). The Arabidopsis GAUT1:GAUT7 complex in suspension cultured cells was further shown to transiently interact with associating proteins including putative methyltransferases [QUASIMODO3 (QUA3) and AT4G18030], KORRIGAN1, and two homologs of mammalian ribophorins I and II (Atmodjo et al., 2011). These findings shed new light on the biosynthetic components involved in the biosynthesis of HG in suspension cultures and leaves, but raise the question of why nature evolved such a complicated mechanism to target GAUT1 to the Golgi apparatus. Moreover, while recovery of gaut 1 homozygous mutants has not been reported, suggesting a lethal or severely deleterious phenotype, gaut7 homozygous mutants have no discernable phenotype, leaving open the question of whether alternative protein anchors exist for GAUT1.
Here we report that, in addition to GAUT7, two other homologs of GAUT1, namely GAUT5 (AT2G30575) and GAUT6 (AT1G06780), can also tether GAUT1 in the Golgi apparatus. Our results reveal that the GAUT1-tethering by GAUT5, GAUT6, and GAUT7 is required in pollen for native pollen tube growth, and that lack of this tethering impacts the synthesis and deposition of highly methylesterified HG at the pollen tube apex, which in turn impacts male fertility. The evolution of this GAUT5, GAUT6, GAUT7 tethering mechanism coincided with the emergence of angiosperms.

\section{MATERIAL AND METHODS}

\section{Plant Materials and Growth Conditions}

Arabidopsis thaliana ecotype Col-0 was used as WT for all experiments. Seeds of T-DNA insertion lines SALK_ 050186 (gaut5-1; located in exon 6), SALK_ 007987 (gaut6-1; located in exon 7) and SALK_015189 (gaut7-1; located in exon 9) were as described in (Caffall et al., 2009). Plants were grown in soil at a $16 \mathrm{~h}$ photoperiod at 100 to $120 \mu \mathrm{E} \mathrm{m}^{-2} \mathrm{~s}^{-1}$, constant temperature of $20^{\circ} \mathrm{C}, 70 \%(\mathrm{v} / \mathrm{v})$ relative humidity and watered as necessary. Stable transformation of WT with the promoter-GUS construct, a negative control construct as well as transformation of the gaut5 $^{-/-}$gaut $^{-1-}$ gaut $^{+/-}$mutant with the GAUT1 chimera construct and a negative control construct was performed using Agrobacterium tumefaciens as the vector through the floral dipping method. BASTA ${ }^{\circledR}$ was used to select for positive transformants, except for the promoter-GUS construct for which kanamycin-based selection was used. Nicotiana benthamiana was grown in soil at a $16 \mathrm{~h}$ photoperiod at 26/ $24^{\circ} \mathrm{C}$ (day/night), 60\% (v/v) humidity and light intensities of 115 to $150 \mu \mathrm{E} \mathrm{m}^{-2} \mathrm{~s}^{-1}$. Reciprocal backcrosses of gaut $^{-1-}$ gaut $^{1-}$ gaut $7^{+/-}$plants to WT were carried out by emasculating stage 12 flowers of the female parent plant and subsequently dabbed with anthers from stage 14 flowers (Smyth et al., 1990) of the male parent. Seeds were collected and progeny from each plant were genotyped by PCR for GAUT5, gaut5, GAUT7 and gaut7 using the genotyping primers in Supplementary Table 2. Transmission of the gaut 7 allele was analyzed by a $\chi^{2}$ test with a significance level of $p<0.05$. To distinguish between the WT cross-pollination and self-pollination, genotyping was done for WT and mutant allele of GAUT5 because the progeny from the crosses are heterozygous in the gaut5 allele, while progeny resulting from self-pollination are homozygous in the gaut5 allele. Three independent attempts to cross the WT (female) with gaut $^{-/-}$gaut6 $^{/-}$gaut $^{+/-}$(male) failed.

\section{In Silico Analysis}

The amino acid sequences of Arabidopsis GAUT1-related family proteins (GAUT1 through GAUT15) were analyzed using the CLC Main Workbench (CLC Bio). Prediction of the transmembrane domain was performing using TMHMM server v. 2.0 (http://www. cbs.dtu.dk/services/TMHMM/). 


\section{Genotyping and RT-PCR Analysis of WT and gaut Mutants}

Genotyping was done using gene- and T-DNA-specific primer sequences obtained from the T-DNA Primer Design tool provided by the Salk Institute Genomic Analysis Laboratory (http://signal. salk.edu/tdnaprimers.2.html); primer sequences are listed in Supplementary Table 2. For RT-PCR analysis, plant materials frozen in liquid nitrogen were ground with a pestle and mRNA extracted using the RNeasy Plant Mini Kit (Qiagen) with oncolumn DNase treatment according to the manufacturer's instruction. The integrity of the RNA was confirmed by gel electrophoresis. Before cDNA synthesis, mRNA concentrations were quantified by NanoDrop (ThermoFisher Scientific) and normalized to the equal amount. cDNA was synthesized using the iScript cDNA synthesis kit (Bio-Rad) according to the manufacturer's protocol. ACTIN2 (ACT2, AT3G18780) was used as a reference gene.

\section{Construction of Fluorescent Protein-Tagged GAUT Proteins and GAUT1 Chimeras}

Coding sequences of GAUT1 and GAUT4 to 7 were amplified by gene specific primers (Supplementary Table 2) and inserted into the $\mathrm{pDONR} / \mathrm{zeo}$ vector by the $\mathrm{BP}$ reaction following the manufacturer's instruction (ThermoFisher Scientific). The coding sequences were subsequently transferred to the GATEWAY Expression plasmid pMDC83 by the LR reaction, leading to the generation of GAUTs fused C-terminally with GFP. GAUT1-YFP was generated using the USER cloning strategy as previously described (Sakuragi et al., 2011a; Sakuragi et al., 2011b). The GAUT1 chimeric proteins XYLT : GAUT1 were generated as follows. The different fragments were PCR amplified in the following combinations: GAUT1(168.673) using the primers GAUT1 C-region fwd and GAUT1 C-region rvs; XYLT was amplified using XYLT CTS fwd and XYLT CTS rvs from Col-0 cDNA (see above). Using USER fusion, the fragments were joined and inserted into the pro35S:USER-eGFP vector (Sakuragi et al., 2011) where the chimeras are controlled by $35 S$ promoter and are fused to GFP in frame. The proGAUT1:USER vector was generated by amplifying the promoter region of GAUT1 using the primers proGAUT1 fwd and proGAUT1 USER rvs, in Supplementary Table 2, and inserting into pCambia3300u by USER fusion. The proGAUT1 USER rvs primer contains a new USER cloning site for the insertion of the chimeras.

\section{Promoter-GUS Constructs}

The promoter regions cloned were: 1400 bp for AtGAUT5, and 1981 bp for AtGAUT6, upstream from the start codon. These were used for construction of transgenic lines bearing the pGAUT5::GUS construct and the pGAUT6::GUS construct by the procedure as described in (Atmodjo et al., 2011).

\section{Subcellular Localization of Fluorescently Tagged GAUT Proteins in N. benthamiana}

The constructs were introduced into Agrobacterium tumefaciens and subsequently into N. benthamiana via Agrobacteria-mediated heterologous transient expression as previously described (Sakuragi et al., 2011a; Sakuragi et al., 2011b). The viral suppressor p19 was included in all experiments. Confocal laser scanning microscopy was carried out using a Leica SP2 or SP5 microscope using laser excitations at $488 \mathrm{~nm}, 514 \mathrm{~nm}$, and $587 \mathrm{~nm}$ for excitation of GFP, YFP, and mCherry, respectively, and emission detections at 495 to $510 \mathrm{~nm}, 560$ to $600 \mathrm{~nm}$, and 600 to $615 \mathrm{~nm}$, respectively, using 20x magnification water objective. The pinhole diameter was set at 1 airy unit. Scan speed was $800 \mathrm{~Hz}$ with a line average of 8 . For identification of the subcellular localization, GAUT-GFP fusion proteins were co-expressed with STtmd-YFP, a Golgi marker protein, as previously described (Sakuragi et al., 2011). Coexpression of GAUT1-YFP with GAUT4-GFP, GAUT5-GFP, and GAUT6-GFP was performed as described (Atmodjo et al., 2011). Co-expression of GAUT1-GFP and GAUT7-YFP was performed alongside as the positive control. Single expression of GAUT4-GFP and GAUT7-YFP was also performed alongside as the negative control. For quantification of GFP and YFP signals upon coexpression, areas with characteristic Golgi morphology were manually selected and the pixel mean value within each area in each of the GFP and YFP detection channels was extracted using Leica LCS Lite software (Leica Microsystems). The values were subsequently plotted using OriginPro (OriginLab Corp.) and ANCOVA was performed using MATLAB (The MathWorks, Inc.).

\section{Yeast-Based Membrane Split-Ubiquitin Assay}

The modified split-ubiquitin assay was performed essentially as previously described (Obrdlik et al., 2004; Lund et al., 2015). Briefly, GAUT coding sequences were PCR amplified using primers detailed in Supplementary Table 2, and ligated into pBT3-N (bait) and pPR3-N (prey) vectors (Dualsystems Biotech AG, Schlieren, Switzerland) at the SfiI restriction site. OstlpNubI, which stably interacts with Cub (Stagljar et al., 1998), was used as the positive control, while the empty NubG vector, pPR3$\mathrm{N}$, is used as the negative control to detect any unwanted autoactivation of baits. Anp1p, a Golgi-localized yeast enzyme involved in N-glycan biosynthesis (Jungmann and Munro, 1998), was fused to Cub and used as the specificity control to determine the degree of random interaction with NubG-fused proteins (preys). The plasmids were introduced in pairs into yeast strain NMY51 by LiAc transformation. Transformants were selected by SD-Leu-Trp and strains carrying both vectors were grown to an $\mathrm{OD}_{546}$ of 1.5. Serial dilutions (between 1- and 1,000 -fold) were spotted on SD-Leu-Trp, SD-Leu-Trp-His and SD-Leu-Trp-His-Ade plates. Growth on the SD-His-Leu-Trp and SD-His-Leu-Trp-Ade plates was scored as an indication of interaction.

\section{Morphological Analysis of Pollen Development and Germination}

Pollen viability was assessed using fluorescein diacetate and Alexander staining as previously described (Alexander, 1969; $\mathrm{Li}, 2011)$. Stage 12 flowers were fixed in Carnoy's solution [60\% $(\mathrm{v} / \mathrm{v})$ ethanol, 30\% (v/v) chloroform, 10\% (v/v) glacial acetic acid] overnight, dissected and the stamens stained in a drop of 
Alexander stain solution [11\% (v/v) ethanol, $0.01 \%(\mathrm{w} / \mathrm{v})$ malachite green, $0.05 \%(\mathrm{w} / \mathrm{v})$ acid fuchsin, $0.005 \%(\mathrm{w} / \mathrm{v})$ orange G, $4 \%(\mathrm{v} / \mathrm{v})$ glacial acetic acid, $25 \%$ glycerol in $\mathrm{ddH}_{2} \mathrm{O}$ ] overnight. Viable pollen grains appear purple, while aborted pollen grains appear green (Alexander, 1969). Pollen germination was examined in vitro adapted from the previously-described method (Rodriguez-Enriquez et al., 2013). Briefly, pollen from stage 13 flowers was placed on the surface of a cellophane membrane lying on a microscope slide with an agarose pad [15\% (w/v) sucrose, $0.01 \% \mathrm{H}_{3} \mathrm{BO}_{3}(\mathrm{w} / \mathrm{v}), 1 \mathrm{mM} \mathrm{CaCl} \cdot 2 \mathrm{H}_{2} \mathrm{O}$ $1 \mathrm{mM} \mathrm{Ca}\left(\mathrm{NO}_{3}\right)_{2} \cdot 4 \mathrm{H}_{2} \mathrm{O}, 1 \mathrm{mM} \mathrm{KCl}, 1 \mathrm{mM} \mathrm{MgCl}_{2}$, and $0.1 \mathrm{mM}$ spermidine, $0.5 \%(\mathrm{w} / \mathrm{v})$ agarose in $\mathrm{ddH}_{2} \mathrm{O}, \mathrm{pH}$ adjusted to 8.0 with $\mathrm{KOH}]$. The slides were incubated in the dark at $24^{\circ} \mathrm{C}$ for 4 hours at $100 \%$ humidity before observation of pollen tube growth using a Leica ICC50 HD microscope (Leica Microsystems, Germany) with $20 \times$ magnification. The length of pollen tubes was determined by using ImageJ (https://imagej.nih.gov/ij/).

\section{Histocytochemistry Analysis}

Flowers from 8-week-old transgenic plants were examined for GUS activity after incubating overnight in a GUS staining solution consisting of $50 \mathrm{mM}$ sodium phosphate buffer, $\mathrm{pH}$ 7.0, $10 \mathrm{mM}$ ethylenediaminetetraacetic acid, $0.1 \%$ (v/v) Triton $\mathrm{X}-100,0.5 \mathrm{mM}$ potassium ferricyanide, $0.5 \mathrm{mM}$ potassium ferrocyanide, and $1 \mathrm{mg} \mathrm{ml}^{-1} 5$-bromo-4-chloro-3-indolyl- $\beta$-Dglucuronide. Chlorophyll and other pigments were removed with $96 \%$ (v/v) ethanol. Multiple T2 plants from at least 5 independent $\mathrm{T} 1$ lines showing a consistent staining pattern for each gene were analyzed.

\section{Immunocytological Analysis of Pollen Tubes and Confocal Microscopy}

The cellophane pads with germinated pollen were immediately submerged in $1 \mathrm{ml}$ of $4 \%$ formaldehyde in PBS (prepared from paraformaldehyde) in six-well microplates and fixed for $15 \mathrm{~min}$. at room temperature. The pads were washed twice with PBS and blocked with 5\% skimmed milk in PBS for $30 \mathrm{~min}$. The blocking solution was exchanged with the primary antibody solution containing JIM7 mAbs (PlantProbes) at 1:10 dilution in blocking solution. After $30 \mathrm{~min}$ incubation the pads were washed twice with PBS and incubated with secondary antibody solution consisting of anti-rat AlexaFluor 555-conjugated antibody (Invitrogen) at 1:300 dilution for $30 \mathrm{~min}$. The pads were washed once with PBS and stained with $\operatorname{COS}^{488}$ probe (Mravec et al., 2014) at 1:500 dilution in $25 \mathrm{mM}$ MES buffer $\mathrm{pH}$ 5.7. After $15 \mathrm{~min}$ incubation, the pads were washed with MES buffer and mounted in a drop of Citifluor (Agar Scientific) on a glass slide and covered by a coverslip. The confocal microscopy was performed on Leica SP5 laser-scanning confocal microscope equipped with Argon (488 nm) and HeliumNeon (555 $\mathrm{nm}$ ) lasers. The pictures were processed with GIMP2 software for overlays and visual enhancement. The signal intensity was measured using ImageJ software.

\section{Herbicide Susceptibility Test}

Arabidopsis seeds were surface sterilized by $10 \%$ (v/v) sodium hypochlorite followed by $70 \%(\mathrm{v} / \mathrm{v})$ ethanol and rinsed five times in sterile water. The sterilized seeds were plated on $1 / 2 \mathrm{MS}$ media containing $1 \%(\mathrm{w} / \mathrm{v})$ sucrose and after vernalization for 2 days in the dark at $4^{\circ} \mathrm{C}$ were allowed to germinate in a growth chamber for 5 days at $22^{\circ} \mathrm{C}$ in the diurnal cycle with the photoperiod of $16 \mathrm{~h}$. Seedlings were transferred to $1 / 2 \mathrm{MS}$ media containing $1 \%$ $(\mathrm{w} / \mathrm{v})$ sucrose and $30 \mu \mathrm{g} \mathrm{ml} \mathrm{m}^{-1}$ phosphinothricine and were incubated for 2 weeks under the same conditions as described above. The numbers of growing and dead seedlings were counted.

\section{Statistical Analysis}

ANOVA (one-way and two-way) and Student's t-test were used. $p$ values $<0.05$ is indicated by different lettering or by ${ }^{*}$.

\section{RESULTS}

\section{GAUT5 and GAUT6 Are Able to Tether GAUT1 in the Golgi Apparatus Similar to GAUT7}

We sought to test the hypothesis that there are additional GAUT1-anchoring proteins that provide complementary functions to GAUT7. We first screened 11 less characterized Arabidopsis GAUT proteins for their ability to localize to the Golgi apparatus by fusing them to green fluorescent protein (GFP), heterologously expressing them in Nicotiana benthamiana, and imaging their expression in the intracellular membrane system by live-cell imaging using confocal laser-scanning microscopy (CLSM) (Sakuragi et al., 2011). We found that GAUT4-GFP, GAUT5-GFP, and GAUT6-GFP showed punctate signals characteristic of the Golgi apparatus (Figure $\mathbf{1}$ and Supplementary Figure 1, in a manner similar to that previously reported for GAUT7 (Atmodjo et al., 2011). The Golgi localization of these constructs was further confirmed by co-localization with the Golgi marker protein STtmd-YFP (Figure 1).

We next tested GAUT4, GAUT5, and GAUT6 for their ability to tether GAUT1 to the Golgi. Arabidopsis GAUT1, due to the Nterminal cleavage at aa 167, cannot by itself localize to the Golgi and is consequently secreted out into the apoplast in the absence of an anchoring protein partner such as GAUT7 (Atmodjo et al., 2011). When transiently expressed in N. benthamiana, GAUT1-GFP did not by itself yield any detectable GFP fluorescence in the apoplast (likely due to the low $\mathrm{pH}$ environment), but did yield punctate Golgi localization signal upon co-expression with GAUT7, as previously reported (Atmodjo et al., 2011). Here we used the same approach, wherein Golgi-retention of GAUT1-YFP by the GAUT candidates was assessed using CLSM upon co-expression of the protein constructs in $N$. benthamiana (Figure 2 and Supplementary Figure 1). Co-expression with either GAUT5-GFP or GAUT6GFP gave rise to punctate GAUT1-YFP signals that overlapped with the GAUT5-GFP or GAUT6-GFP signals (Figures 2B, C). In contrast, co-expression with GAUT4-GFP did not give rise to GAUT1-YFP signals, although punctate GAUT4-GFP signals were clearly visible (Figure 2A). Because GFP and YFP have partially overlapping emission wavelengths, it is possible that GFP signals might have crossed over into the YFP channel. To address this, pixel signal intensities in both the YFP and GFP detection channels were obtained for each of the punctate signals and 

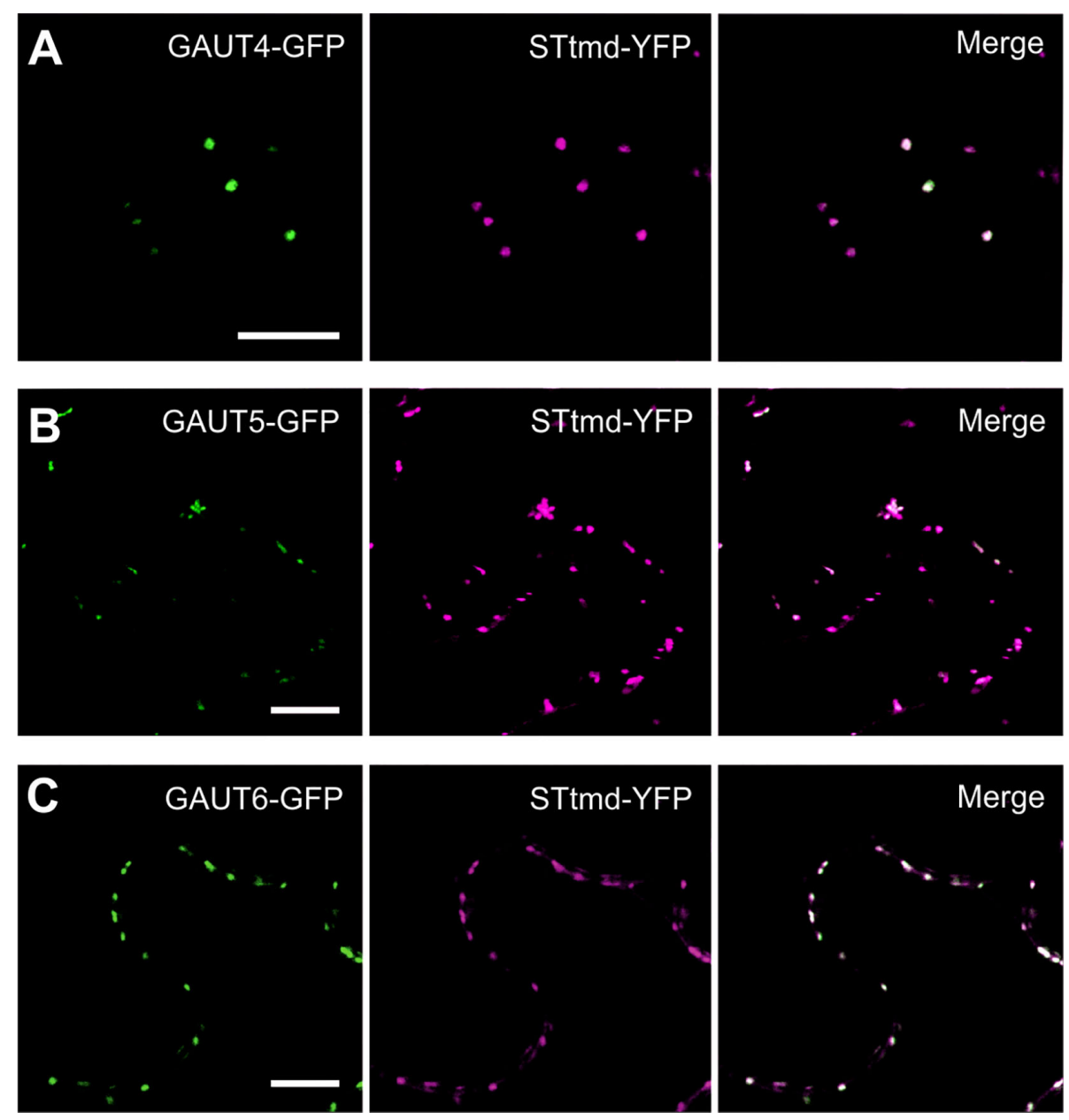

FIGURE 1 | Golgi localization of GAUT4, GAUT5, and GAUT6 GFP fusion cosnstructs. GAUT4-GFP (A), GAUT5-GFP (B), and GAUT6-GFP (C) under the control of 35 S promoter have been co-expressed with the Golgi marker STtmd-YFP (middle row panels) in N. benthamiana. Merge images indicate co-localization (last row panels). Confocal laser scanning microscopy analysis was performed 3 days after transfection. Scale bars $=10 \mu \mathrm{m}$.

compared to those of the GAUT4-GFP alone, which was used as a control for punctate GFP signals (Atmodjo et al., 2011; Figures 2E-H). Signals derived from the GAUT4-GFP control largely distributed along the $x$ axis representing GFP signals, indicating limited signal bleed-through between the GFP and YFP detection channels. Signals derived from co-expression of GAUT1-YFP with GAUT4GFP distributed similarly to the GAUT4-GFP control, which is supported by analysis of co-variance (ANCOVA, $p>0.05$ ) (Figure 2E). In contrast, the signals derived from co-expression of GAUT1YFP with either GAUT5-GFP or GAUT6-GFP displayed notably different distributions from that of the GAUT4-GFP control (Figures 2F, G), more resembling the distribution of GAUT1YFP and GAUT7-GFP expression (Figure 1H). Moreover, the differences between the samples and the GAUT4-GFP control were statistically significantly different (ANCOVA, $p<0.05$ ). Taken together these results indicate that unlike GAUT4, GAUT5, and GAUT6 were able to influence the GAUT1 trafficking and to retain GAUT1 in the Golgi apparatus in planta in a manner similar to that previously reported for GAUT7 (Atmodjo et al., 2011).
To further investigate protein-protein interactions between GAUT1 and GAUT5, GAUT6, and GAUT7, we used a modified yeast-based split-ubiquitin system (Lund et al., 2015) (Figure 2I). GAUT1 was N-terminally fused with a reporter consisting of a synthetic transcriptional factor linked to the C-terminal fragment of ubiquitin (TF-Cub) to generate a bait, while GAUT1, GAUT5, and GAUT6, alongside GAUT7 were Nterminally fused with the N-terminal fragment (NubG) of an ubiquitin version bearing an amino acid substitution that prevents irreversible reconstitution of TF-Cub and NubG. Control experiments revealed that TF-Cub-GAUT1 and TFCub-GAUT7 proteins were functional because they complemented the yeast growth with the positive control NubI. Further control experiments revealed that NubG-fused GAUT proteins did not promiscuously interact with Anp1p, a yeast Golgi-resident type-II membrane protein, as no complementation of growth was observed. Co-expression of TF-Cub-GAUT1 with NubG-fused GAUTs resulted in complementation in all tested combinations, indicating that 

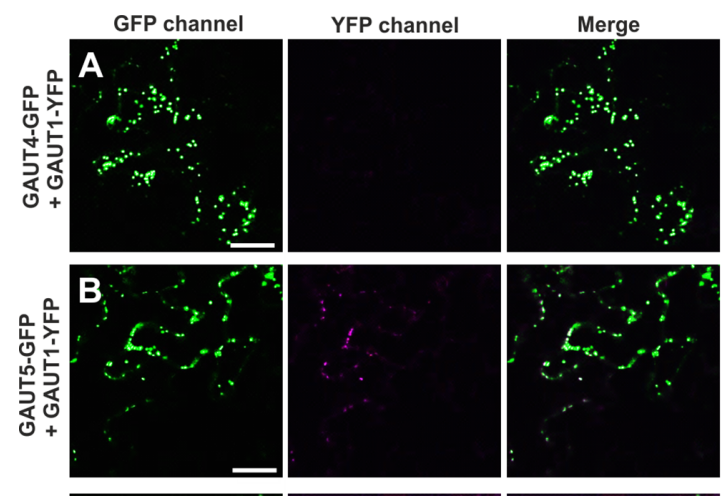

\section{E $^{100}$ 1 O GAUT1-YFP+GAUT4-GFP \\ $\square$ GAUT4-GFP (ANCOVA: $p>0.05$ )}
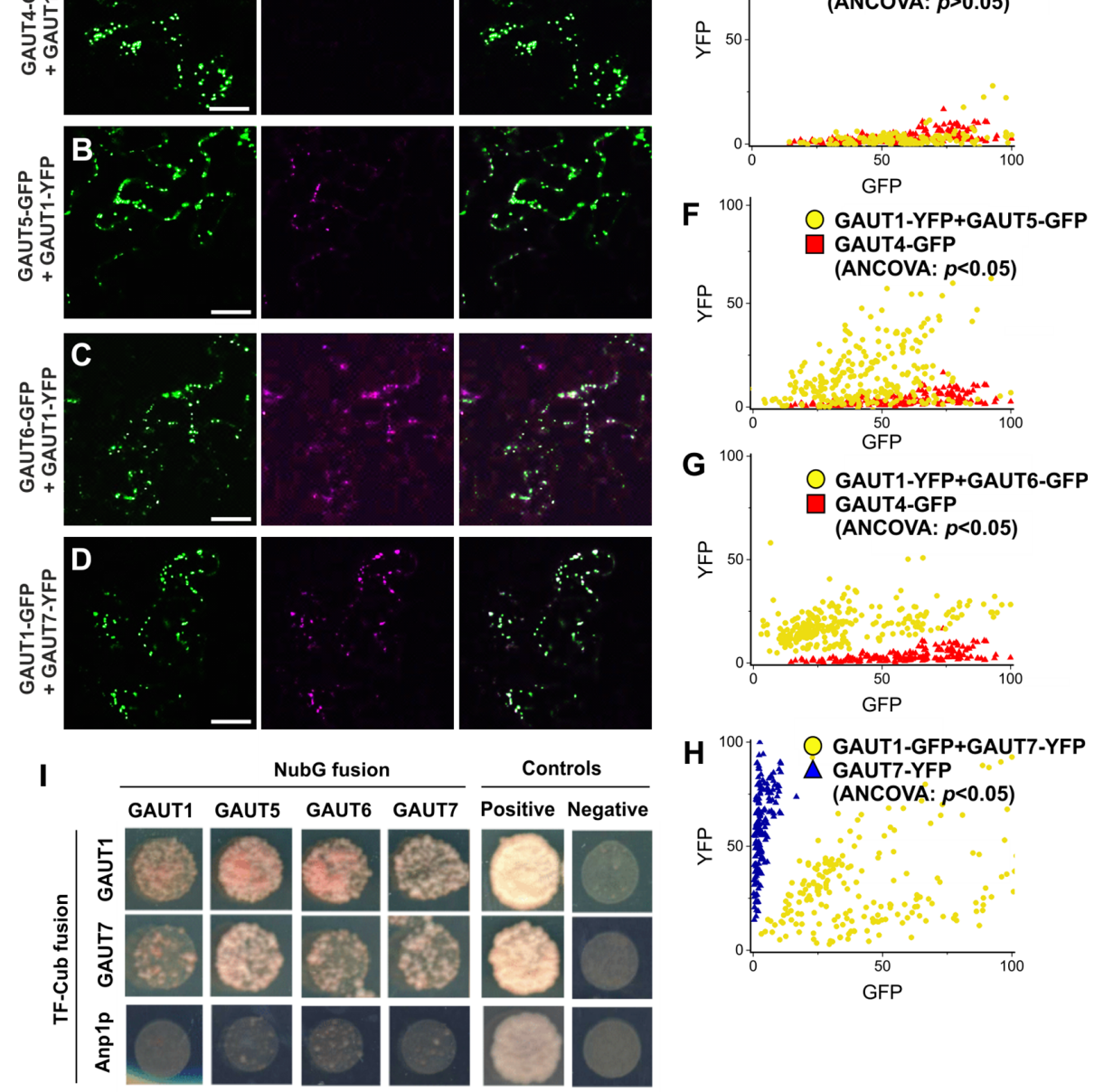

FIGURE 2 | Tethering of GAUT1 by GAUT5, GAUT6, and GAUT7 in the plant Golgi. (A-D) Coexpression analysis: GAUT1-YFP co-expressed with (A) GAUT4-GFP, (B) GAUT5-GFP, (C) GAUT6-GFP, and (D) GAUT1-GFP co-expressed with GAUT7-YFP as a positive control. Expression of these proteins was performed transiently in N. benthamiana. Confocal laser scanning microscopy was performed 3 days after transfection. Scale bars = $10 \mu \mathrm{m}$. (E-H) Quantifications of GFP and YFP signals (in relative range from 0 to 100, max. signal) in individual punctate signals: GAUT1-YFP co-expressed with (E) GAUT4-GFP ( $\mathrm{n}=153$ ), (F) GAUT5-GFP ( $\mathrm{n}=153$ ), (G) GAUT6-GFP ( $\mathrm{n}=262$ ), and (H) GAUT1-GFP co-expressed with GAUT7-YFP as a positive control ( $\mathrm{n}=195)$. Confocal laser scanning microscopy was performed 3 days after transfection. $p$ values based on ANCOVA are shown. (I) Protein-protein interaction assay by yeast-based membrane split-ubiquitin complementation. The yeast growth on the SD-His-Leu-Trp-Ade drop out medium indicates interaction. The positive and negative controls are Nubl and pPR3-N, respectively. The Golgi-bound alpha-1,6-mannosyltransferase (Anp1p) was used as specificity control.

GAUT1 interacts with GAUT5, GAUT6, and GAUT7, and also with itself (Atmodjo et al., 2011; Amos et al., 2018). Coexpression of TF-Cub-GAUT7 constructs with NubG-fused GAUTs also resulted in yeast growth in all tested combinations, indicating that GAUT7 can interact with GAUT5 and GAUT6 along with GAUT1 and itself (Atmodjo et al., 2011). The results show that GAUT5 and GAUT6 function similarly to GAUT7 in protein-protein interaction and in retaining GAUT1 in the Golgi apparatus, and thus could compensate for the loss of GAUT7 function in gaut7 mutant showing no obvious phenotype (Caffall et al., 2009).

\section{Three GAUT1-anchoring Proteins (GAUT5, GAUT6, GAUT7) Are Required for Male Fertility}

The existence of three GAUTs that could serve as anchoring proteins for the HG biosynthetic GAUT1 led us to propose three testable hypotheses for their function. (i) All three GAUTs (GAUT5, GAUT6, GAUT7) are genetically redundant. (ii) Each GAUT has a unique function, and thus, knockout expression of any the GAUTs would lead to a phenotype. (ii) Each GAUT has a unique function in vivo, but this function can be partially complemented by the other GAUTs. 
To test these hypotheses, we analyzed the T-DNA insertion mutants of these three genes. Both the gaut5-1 and gaut6-1 lines have been previously reported as homozygous knock-out mutants with T-DNA insertions in exons (Caffall et al., 2009). These mutants were confirmed by PCR genotyping and by RTPCR transcript analysis (Figures 3A, B and Supplementary Figure 3). The gaut7-1 homozygous T-DNA insertion line, with an insertion in the $9^{\text {th }}$ exon (Figures $3 \mathbf{A}, \mathbf{B}$ ), was previously considered a knock-down mutant because a reduced amount of the GAUT7 transcript was still detected when a set of oligonucleotide primers that bind upstream of the T-DNA insertion site was used (Caffall and Mohnen, 2009). However, our RT-PCR analysis using a set of primers that bind sequences flanking the T-DNA insertional site did not generate any detectable PCR product in gaut7-1 (Figure 3C). Moreover, in silico sequence analysis revealed that the insertion of the T-DNA cassette in the gaut7-1 line created a stop codon, which would result in a truncated GAUT7 protein consisting of the Nterminal 372 residues out of 674 residues and missing two conserved motifs amongst all GAUT1 family proteins, namely DxD (amino acid positions between 450 and 452) and HxxGxxKPW (amino acid positions between 581 and 589) (Supplementary Figure 2). These motifs are expected to coordinate $\mathrm{Mn}^{2+}$ and interact with the NDP-sugar donor, respectively (Sterling et al., 2006; Yin et al., 2010a). From these analyses, we concluded that gaut7-1 is likely a null mutant.

When grown under the standard growth conditions, single mutants gaut5-1, gaut6-1, and gaut7-1 (hereafter in this paper referred to as gaut5, gaut6, and gaut7) grew similarly to WT (Figures 3D, E) as previously reported (Caffall et al., 2009). These results showed that each of the three genes is dispensable for the viability of Arabidopsis, which is in contrast to GAUT1 for which no homozygous mutants have yet been reported (Yin et al., 2010b). The lack of a growth phenotype in gaut5, gaut6, and gaut7 homozygous mutants supported hypothesis (i), indicating that at least one of the three GAUTs functions redundantly with regards to the overall plant growth.

To further probe whether GAUT5, GAUT6, and GAUT7 had unique physiological roles in planta, double mutants of gaut5, gaut6, and gaut7 in all combinations were generated by crossing fertile individual single mutants. All the recovered double mutants gaut5gaut6, gaut5gaut7, and gaut6gaut7 plants grew normally and were fertile, similar to WT plants (Figures 3D, E). This demonstrated that a single functional copy of GAUT5 or GAUT6 or GAUT7 was sufficient for normal plant growth and fertility under greenhouse conditions, further supporting hypothesis (i). For genetic analyses, the heterozygous parental lines gaut $5^{+/-}$gaut $^{/-}$, gaut $5^{+/}$gaut $7^{-1}$, and gaut $^{\text {/- }}$ gaut $^{+/-}$ were obtained and segregation of the respective heterologous alleles was monitored. The Mendelian segregation ratio of 1 (WT): $2\left(\right.$ gaut $^{+-}$gaut $\left.^{1-}\right): 1$ gaut $^{-/-}$gaut $\left.^{-1-}\right)$ and 1 (WT): 2 $\left(\right.$ gaut $^{+/-}$gaut $\left.^{-1-}\right): 1\left(\right.$ gaut $5^{-/-}$gaut $\left.7^{--}\right)$indicated that GAUT7 could fully compensate for the function(s) of GAUT5 and GAUT6 and that GAUT6 could likewise fully compensate for the function of GAUT5 and GAUT7, respectively, in fertilization. Interestingly, however, segregation of gaut6 ${ }^{-1-}$ gaut $7^{+/-}$was skewed with a ratio of approximately 4 (WT): 6 $\left(\right.$ gaut $^{-1-}$ gaut $\left.^{+-}\right)$: 1 (gaut6 $^{-1-}$ gaut $^{-1}$ ) (Table 1), suggesting that GAUT6 and/or GAUT7 had some fertilization-related functions for which GAUT5 could not complement.

Efforts to generate the triple mutant failed to yield the triple homozygous gaut5 gaut6 gaut7 mutant, indicating that the presence of at least one of the three genes is necessary for fertility. A heterozygous gaut $5^{-1-}$ gaut $^{/-}$gaut $^{+/-}$mutant, however, was able to be isolated and this mutant appeared indistinguishable from WT upon maturity (Figures 3D, E). Selfing of this mutant gave a highly-skewed segregation pattern of the gaut $^{+/-}$alleles in the ratio of 1 (WT): 1 (gaut $\left.7^{+/-}\right): 0$ $\left(\right.$ gaut $7^{-1}$ ) (Table 1). This segregation pattern is characteristic of defective transmission of the mutant allele through either the female or male gametophyte (Rédei, 1965).

To ascertain the nature of the gametophyte defect, crossfertilization was performed between the gaut $5^{-1-}$ gaut $^{-1-}$ gaut $7^{+-}$mutant and WT. When the ovules of the heterozygous triple mutant plants were cross-fertilized with pollen from WT plants, the offspring population showed an expected normal segregation ratio $1\left(\right.$ gaut $\left.7^{+}\right)$: 1 (gaut $7^{-}$) alleles (Table 1), indicating that the female gametophytic transmission in the heterozygous triple mutant is functional. In contrast, when the ovules of WT plants were crossed with pollen from the heterozygous triple mutant, no seed was recovered even after several independent attempts, indicating that male gametophytic transmission through both gaut $5^{-}$gaut $\sigma^{-}$gaut $7^{-}$and gaut5 gaut $\sigma^{-}$gaut $7^{+}$pollen is inhibited during cross pollination, and thus, confirming a role in male fertility. That no seeds were recovered was unexpected, considering that selfed gaut $5^{-1-}$ gaut6 $^{-1-}$ gaut $7^{+/-}$mutant could still produce seeds as mentioned above (Table 1). We speculate that, unlike during native selfing when transmission of paternal gaut $5^{-}$gaut 6 gaut $7^{+}$ allelic combination is functional, during crosspollination external stress and dosage dependent effects of the heterozygous gaut $7^{+/}$ genotype in the paternal tissues and/or premeiotic microsporocytes affect pollination. Monosaccharide compositions of cell walls isolated from rosette leaves from wild type and the different mutants revealed no differences in most sugars between WT and all the homozygous single, homozygous double, and gaut $5^{-1-}$ gaut $\sigma^{1-}$ gaut $7^{+/-}$triple mutant (Supplementary Figure 4). The only exceptions were a slightly elevated GalA content in the gaut5 single mutant, a concomitant reduction in the Glu and Gal content, and a slight elevation in the Xyl+Man content in gaut7, gaut5 gaut6, gaut5 gaut7, gaut6 gaut7, and gaut $5^{-1-}$ gaut $^{-1-}$ gaut $7^{+/-}$ mutants. Taken together the combined results suggested that although they might have redundant functions in vegetative tissue, the three GAUTs either had unique functions or could partially complement for each other during fertilization in the reproductive tissues, thus supporting hypothesis (ii) or (iii).

\section{GAUT5, GAUT6, and GAUT7 Are Expressed in Pollen}

Consistent with the defects in the male transmission, the GAUT6 and GAUT7 transcripts have been detected in mature pollen as well as in pollen tubes by Arabidopsis ATH1 microarray analysis 
A

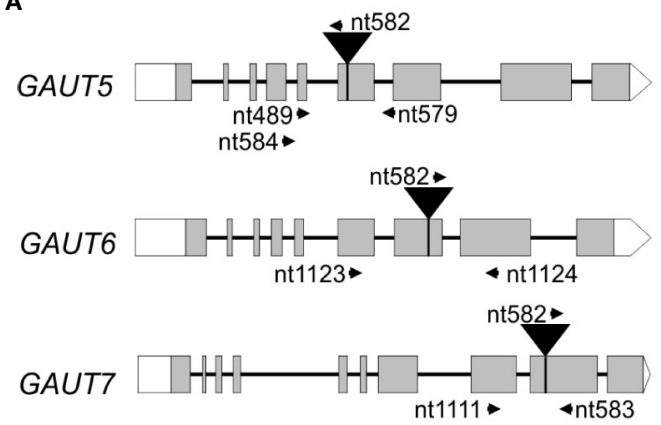

C
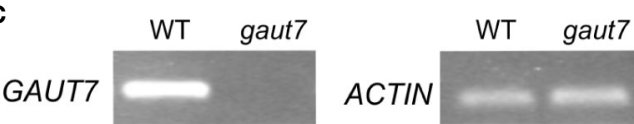

B

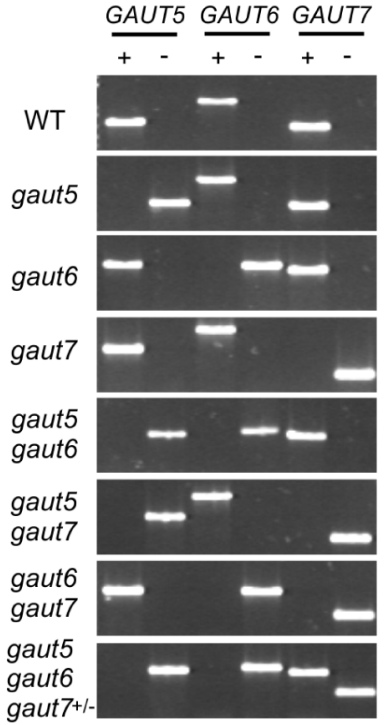

D

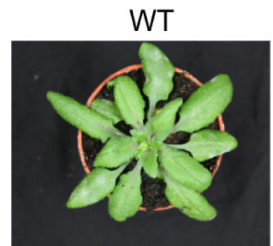

gaut5 gaut6

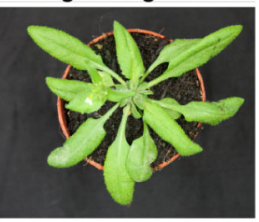

gaut5

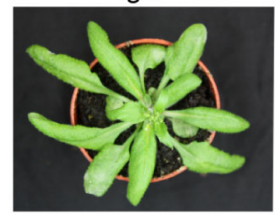

gaut5 gaut7

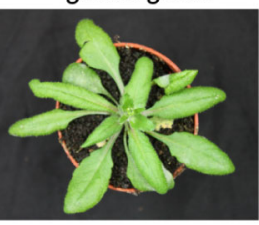

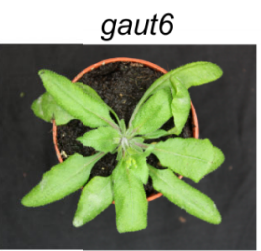

gaut6 gaut7

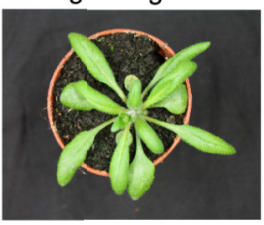

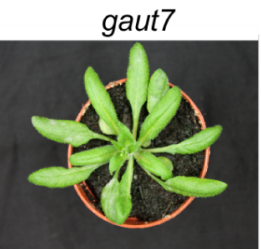

gaut5 gaut6 gaut7+/-

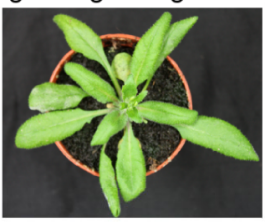

E

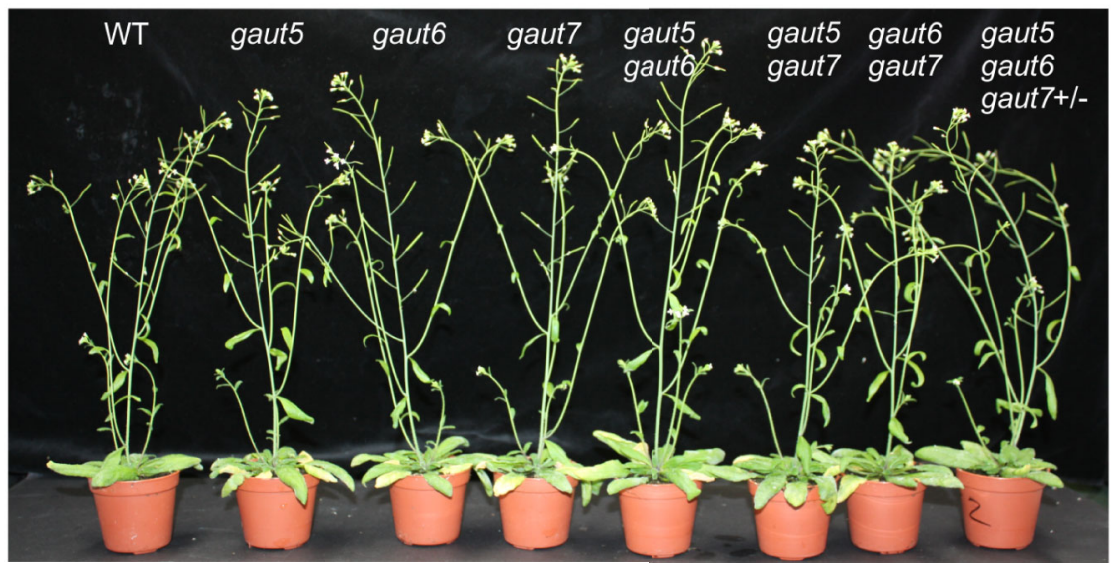

FIGURE 3 | Genotyping and macroscopic phenotypes of the gaut single, double, and triple mutants. (A) Gene models. T-DNA insertion sites are indicated as black triangle inserts and primer binding sites as black arrows. The sequences of the primers are found in Supplementary Table 2. (B) PCR-based genotyping. PCR reactions using primer sets targeting the WT loci are indicated as "+," where those targeting the mutant loci are indicated as "-." (C) GAUT7 transcript analysis by RT-PCR for WT and gaut7-1 homozygous plants. Total RNA was isolated from rosette leaves and equal amounts of RNA were used as the template. RT-PCR of the ACTIN transcript is shown as a positive control. (D) Images of fully expanded rosettes from 8-week-old plants. (E) Images of whole 14-week-old plants. 
TABLE 1 | Genetic segregation analysis of gaut mutants.

\begin{tabular}{|c|c|c|c|c|}
\hline \multirow{2}{*}{$\begin{array}{l}\text { Parents } \\
\text { Self fertilization }\end{array}$} & \multirow[t]{2}{*}{$n$} & \multicolumn{3}{|c|}{ Offspring genotypes [observed (expected Mendelian)] } \\
\hline & & WT & gaut5 $^{+/-}$ & gaut5 $^{-/-}$ \\
\hline gaut5 $^{+/-}$gaut6 $^{-/-}$ & 45 & $9(11.25)$ & $26(22.5)$ & $10(11.25)$ \\
\hline \multirow[t]{2}{*}{ gaut5 $5^{+/-}$gaut $7^{-/-}$} & 90 & $30(22.5)$ & $39(45)$ & $21(22.5)$ \\
\hline & & WT & gaut $^{+/-}$ & gaut $^{-/-}$ \\
\hline gaut6 $^{-/-}$gaut $^{+/-}$ & 102 & $39(27)$ & $54(54)$ & $9(27)^{\star}$ \\
\hline gaut5 $^{-/-}$gaut6 $^{-/-}$gaut $^{+/-}$ & 96 & $50(24)$ & $46(48)$ & $0(24)^{\star}$ \\
\hline Cross fertilization & & WT & gaut $^{+/-}$ & \\
\hline $\begin{array}{l}\text { WT }(\text { pollen }) \times \\
\text { gaut5 } 5^{-/-} \text {gaut6 }{ }^{-/-} \text {gaut } 7^{+/-} \text {(ovules) }\end{array}$ & 31 & $15(15.5)$ & $16(15.5)$ & \\
\hline T2 population of chimera pGAUT1 ::XYLT : GAUT1 in & $n$ & \multicolumn{3}{|c|}{ Offspring genotypes [observed (expected if rescue)] } \\
\hline gaut5 $^{-/-}$gaut6 $^{-/-}$gaut $^{+/-}$ & & WT & gaut $7^{+/-}$ & gaut7 $7^{-/-}$ \\
\hline line 897-10 & 68 & $22(20.4)$ & $24(34)$ & $22(13.6)$ \\
\hline line 897-14 & 65 & $20(21.3)$ & $29(35.5)$ & $22(14.2)$ \\
\hline empty vector control & 63 & $30(18.6)$ & $32(31)$ & $0(12.4)$ * \\
\hline
\end{tabular}

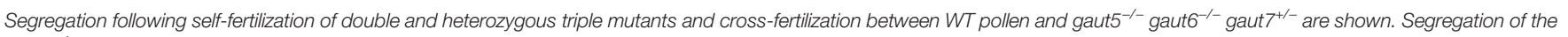

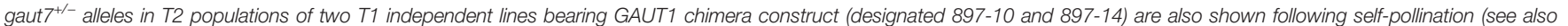

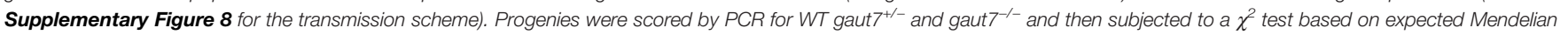

(Wang et al., 2008; Qin et al., 2009). While the GAUT5 transcript was not analyzed in the microarray due to the lack of probes against this transcript, an mRNA sequencing study of mature pollen revealed that GAUT5 transcript is also present (Loraine et al., 2013). Moreover, proteomic analyses of pollen have detected the presence of GAUT1, GAUT5, GAUT6, and GAUT7 proteins (Grobei et al., 2009; Pep2Pro, http://fgczpep2pro.uzh.ch/). We also analyzed the promoter activity of the four GAUT genes using promoter-GUS constructs (Figure 4). GUS staining revealed partially overlapping patterns in flowers. While the promoter activity of GAUT1 was prominent in several tissues including petals, anthers, anther filaments, stigma and pollen, those of GAUT5, GAUT6, and GAUT7 were prominently seen in anther and specifically in the pollen grains (Figures 4A-H). The transcript and protein expression profiles are therefore consistent with a role of these GAUTs in pollen formation and/or function.

\section{GAUT5, GAUT6, and GAUT7 Are Required for Pollen Germination and Elongation}

The results from genetic analyses led us to investigate the pollen. We first tested the viability of pollen in the anthers. Staining with fluorescein diacetate $(\mathrm{Li}, 2011)$ showed the same level of staining in pollen grains from WT and in pollen from heterozygous triple gaut5 gaut6 gaut $7^{+/-}$mutant plants (Supplementary Figure 5A). Alexander staining (Alexander, 1969; Robertson et al., 2004) also yielded a purple colorization of both WT and gaut5 $5^{-/-}$gaut $\sigma^{/-}$ gaut $7^{+-}$mutant-derived pollen (Supplementary Figure 5B). These results confirmed that the pollen heterozygous triple mutant is comparably viable as that of WT.

We next studied pollen germination and pollen tube growth in vitro as previously described (Rodriguez-Enriquez et al., 2013). Pollen from newly opening flowers of all genotypes were grown simultaneously in a temperature and humidity-controlled growth chamber and analyzed. The WT and gaut $5^{-1-}$ gaut ${ }^{1-}$ gaut $7^{+/-}$mutant displayed significant differences in in vitro pollen germination (Figure 5A). A large proportion ( 60\%) of the heterozygous triple mutant pollen did not germinate and nearly half of those that did germinate displayed bursting tubes. On the contrary, almost $80 \%$ of WT pollen successfully germinated and only $5 \%$ of these showed burst pollen tubes. Some of the single and double homozygous mutants displayed intermediate levels of pollen germination. While the gaut5 and gaut6 single mutants displayed minor, non-significant increases in the proportion of non-germinating pollen, the gaut7 single mutant displayed a significant increase in the number of nongerminating pollen grains. Furthermore, all the double mutants displayed additive effects of their respective single mutants (Figure 5A). These results indicate that GAUT7 is required for WT-like germination and that GAUT6 and GAUT5 also function in pollen germination. In contrast to the gaut5 $5^{-1-}$ gaut $^{-/-}$gaut $7^{+/-}$triple mutant, the proportion of bursting pollen tubes was not altered in the single and double homozygous mutants compared to WT, supporting some level of redundancy in GAUT5, GAUT6, and GAUT7 function in maintaining pollen tube strength.

Measurement of pollen tube length after 4 hours of germination revealed marked differences across the genotypes (Figure 5B). The mean length of the WT pollen was $290 \mu \mathrm{m}$. While the mean length of the gaut6 mutant pollen tube was indistinguishable from that of WT, those of gaut5 and gaut7 mutants were significantly shorter $(220 \mu \mathrm{m}$ and $210 \mu \mathrm{m}$, respectively; $p<0.05$ ), suggesting a role of GAUT5 and GAUT7 in pollen tube elongation. All the double mutants and the heterozygous triple mutants also had significantly shorter pollen tubes $(\sim 210 \mu \mathrm{m})$ than WT $(p<0.05)$. Taken all together, these results demonstrated that the absence of GAUT5, GAUT6, and GAUT7 significantly impaired the germination of pollen and subsequent growth of the pollen tube. This was especially severe in the gaut $5^{-/-}$gaut $^{/-}$gaut $7^{+-}$mutant that consequently resulted in male infertility. GAUT5, GAUT6, and GAUT7 operate in the deposition of $\mathrm{HG}$ in the pollen tube cell wall. 

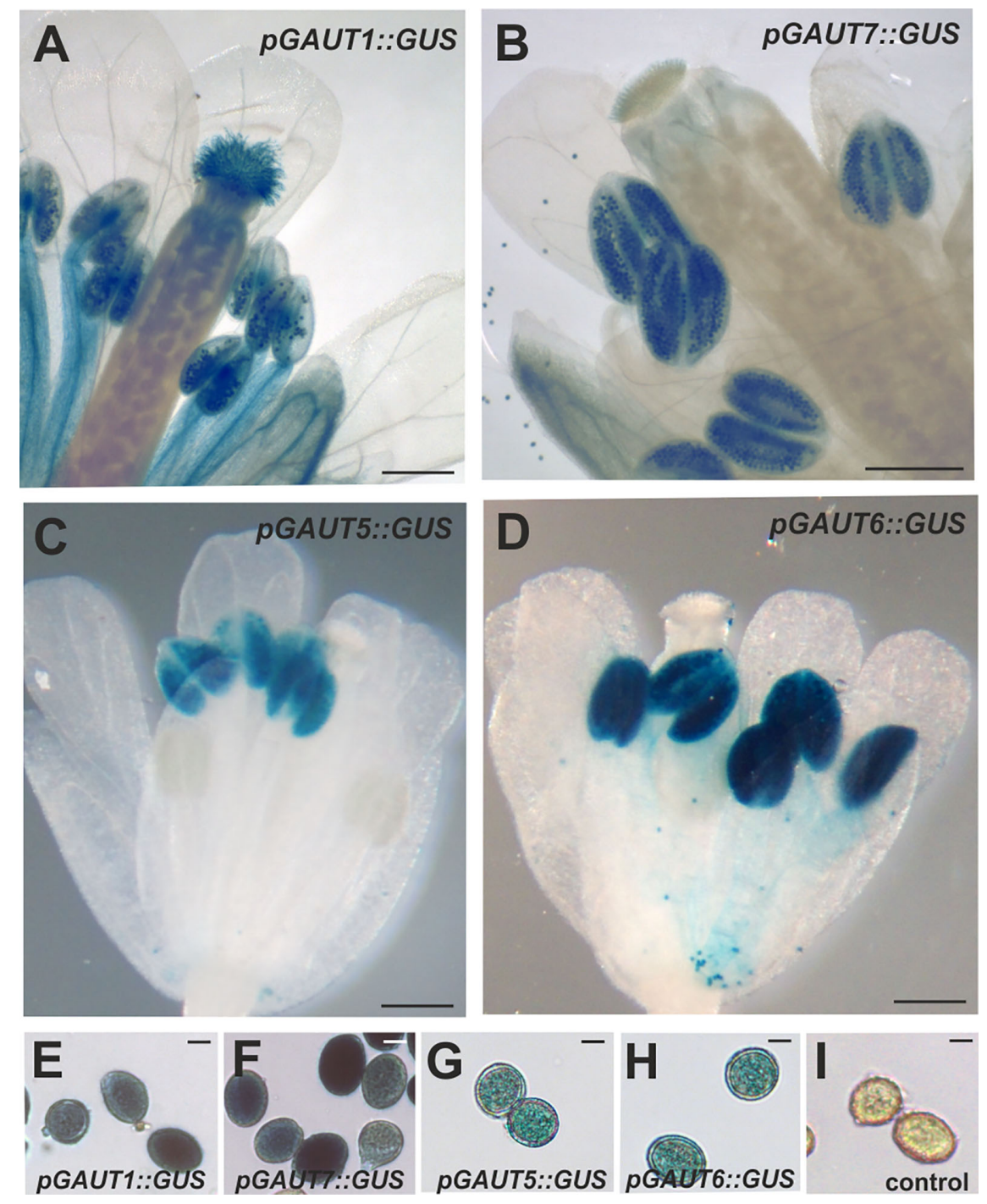

FIGURE 4 | Activity of GAUT1, GAUT5, GAUT6, and GAUT7 promoters in flowers. WT plants were transformed with the genomic regions upstream of the GAUT5 gene and the GAUT6 gene fused with the $\beta$-glucuronidase (GUS) gene and T2 plants were analyzed as described in Materials and Methods. (A-D) GUS staining of the whole flowers, of pGAUT1::GUS (A), pGAUT7::GUS (Atmodjo et al., 2011) (B), pGAUT5::GUS (C) and pGAUT6::GUS (D). Scale bars = 1 mm. (E-I) GUS activity in pollen of pGAUT1::GUS (E), pGAUT7::GUS (F), pGAUT5::GUS (G) and pGAUT6::GUS line (H). (I) Pollen from a negative control plant bearing the empty GUS vector. Scale bars $=10 \mu \mathrm{m}$.

In order to investigate the roles of GAUT5, GAUT6, and GAUT7 in HG synthesis in pollen tubes, we performed immunolocalization using a monoclonal antibody (mAb) JIM7 that recognizes HG with a high degree of esterification-the form of HG synthesized in the Golgi and secreted at the tip of the pollen tube (Chebli et al., 2012; Figure 5C and Supplementary Figure 6). Quantification of the $\mathrm{mAb}$-generated signals from the tip and the shaft region of the growing pollen tube (Figure 5D) showed that in the case of the gaut5, gaut6, and gaut7 single mutants, the signal was reduced compared to WT, although to a different extent among the different gaut mutants. In the case of gaut7, the signal was visibly mislocalized with more equally distributed signal along the whole pollen tube (Supplementary
Figure 6). In comparison, the gaut5 mutant retained JIM7 signal primarily at the tip with much reduced signal on the shaft, while the gaut6 mutant had overall similar, but slightly reduced, tip to shaft staining as the WT. These results suggest that the different GAUTs may affect the amount and location of methylesterified HG in the growing pollen tube. JIM7 binding at the tip and the shaft was almost completely abolished in all double and gaut $5^{-1-}$ gaut $^{-/-}$gaut $7^{+/-}$triple mutant (Supplementary Figure 6), demonstrating the critical importance of GAUT5, GAUT6, and GAUT7 in anchoring GAUT1 for synthesis of methyl-esterified HG in growing pollen tubes. Altered fluorescent intensity and distribution were also observed in the double mutants labeled with $\operatorname{COS}^{488}$ (chitosan oligosaccharides aminooxy-functionalized 

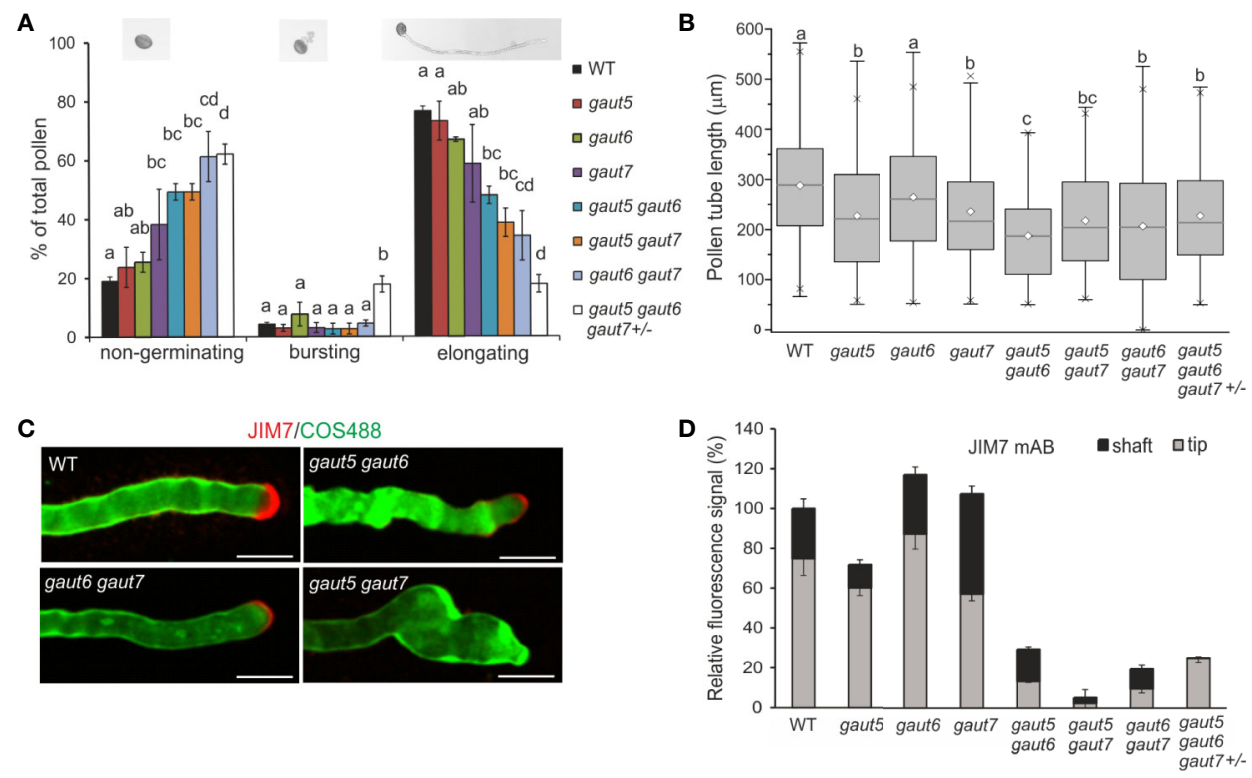

FIGURE 5 | Pollen tube germination and elongation of the gaut5, gaut6, gaut7 homozygous single, homozygous double, and triple mutants (gaut5 ${ }^{-/-}$gaut6 ${ }^{-/-}$ gaut $7^{+/-}$). (A) Distributions of pollen germination phenotypes: "non-germinating" pollen, "bursting" pollen, and pollen with "elongating" tubes ( $\left.\mathrm{n}>100\right)$. Different letters indicate significant differences as determined by One-way ANOVA $(p<0.05)$. (B) The pollen tube lengths were measured in vitro following 4 hours of pollen germination ( $\mathrm{n}>100$ ). (C) Double labeling of lowly methylesterified HG using the $\mathrm{COS}^{488}$ probe (green) and of highly methylesterified HG with JIM7 mAb (red). Instances of complete lack of JIM7 was visible for gaut5 gaut7 double mutant. Scale bars = $10 \mu \mathrm{m}$. (D) Quantification of the signals after labeling HG in the pollen tip and shaft regions with JIM7 ( $n \geq 5$, SEM), expressed as relative to the level in the WT pollen tip.

with Alexa Fluor fluorophore; Mravec et al., 2014), which is a probe specific for $\mathrm{HG}$ with a low degree of esterification (similar to the commonly used JIM5 mAb) and labels the subapical and shaft region of WT pollen tubes (Figure 5C). Taken together, the results indicate that the GAUT1-tethering proteins are required for normal synthesis and deposition of methylesterified HG at the pollen apex and that their loss affects the distribution of methylesterified HG along the pollen tube shaft in GAUT homolog-specific manners. These results support hypothesis III and indicate that each GAUT has a unique function that is partially complemented by the other GAUTs.

\section{A Chimeric, Golgi-Localized GAUT1 Restores Male Fertility in the Heterozygous Triple gaut Mutant}

Based on the previous results we hypothesized that the severe pollen-related phenotypes were caused by mislocalization of GAUT1 due to the absence of the three anchoring GAUTs (GAUT5, GAUT6, GAUT7), resulting in altered HG synthesis. To test this, we attempted to trace the localization of GAUT1 Cterminally fused with mCherry in the series of the gaut mutants. In N. benthamiana, GAUT1-mCherry localized to the cell periphery (Supplementary Figure 7). These results suggest that the endogenous leaf-expressed GAUTs of tobacco are not able to tether the introduced Arabidopsis GAUT1-mCherry to the Golgi apparatus. Furthermore, the localization of GAUT1mCherry in Arabidopsis under the control of the native GAUT1 promoter failed due to insufficient signals above the noise, most likely because of low levels of native GAUT1expression. We thus took an alternative approach by creating a GAUT1 construct capable of accumulating in the Golgi apparatus independently of GAUT5, GAUT6, and GAUT7 and asked whether expression of such a construct would enable recovery of homozygous triple mutant gaut5 gaut6 gaut7 plants. To accomplish this, the Cterminal region of GAUT1 (amino acid positions between 168 and 673) containing the presumed catalytic domain was fused with the $\mathrm{N}$-terminal sequence (amino acid positions between 1 and 90) of a $\beta$-1,2-xylosyltransferase (XYLT, At5g55500), a glycosyltransferase involved in $\mathrm{N}$-glycosylation (Figure 6A). This N-terminal sequence was previously shown to be sufficient for localization in the medial Golgi cisternae (Schoberer et al., 2013). The subcellular localization of the chimeric GAUT1 was analyzed by heterologous expression in N. benthamiana using a $35 \mathrm{~S}$ promoter to drive its expression (Figure 6B). Punctate fluorescence signals were observed for the chimeric GAUT1 construct which co-localized with the Golgi marker STtmd-YFP (Munro, 1995; Saint-Jore-Dupas et al., 2004; Sakuragi et al., 2011). These results show that the chimeric GAUT1 is able to localize to the Golgi apparatus.

To test whether the chimeric GAUT1 protein was able to rescue the phenotype of multiple gaut mutant pollen, we created a construct where the chimeric GAUT1 was expressed from the native GAUT1 promoter. These constructs were introduced into the Arabidopsis gaut $^{-/-}$gaut $^{/-}$gaut $7^{+-}$mutant via stable 
A

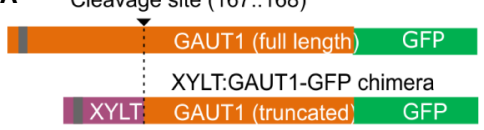

B

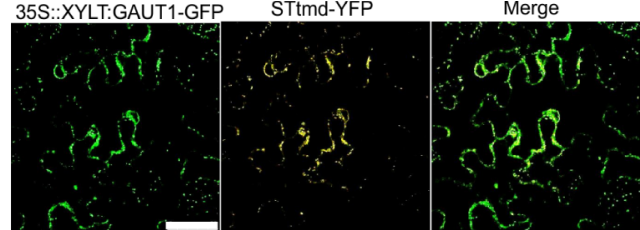

C

-WT $\quad$ Empty vector $\square$ pGAUT1::XYLT:GAUT1 (line 897-10)

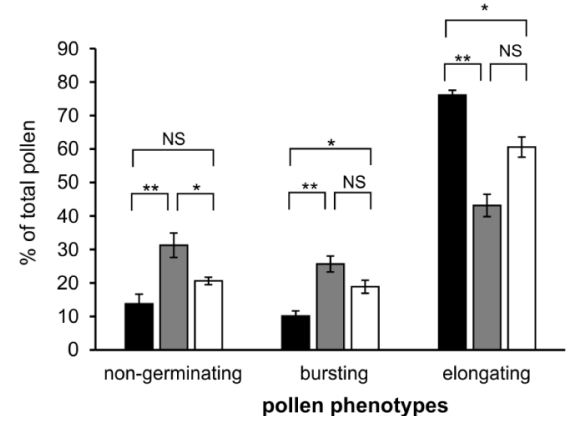

D $\square$ WT $\square$ Empty vector $\square$ pGAUT1::XYLT:GAUT1

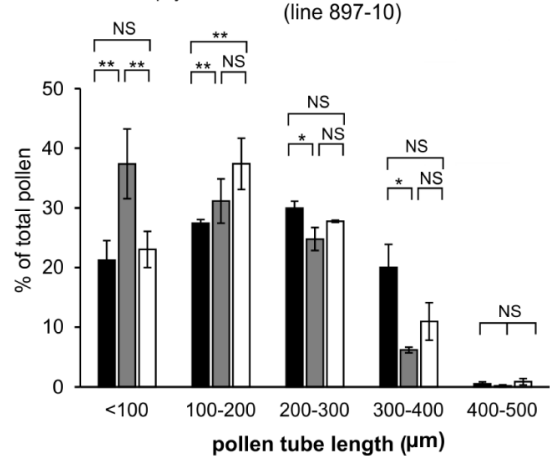

FIGURE 6 | The pGAUT1::XYLT : GAUT1 chimera restores pollen tube elongation in the gaut5 gaut6 gaut $7^{+/-}$mutant. (A) Design of the GAUT1 chimera construct. The assumed proteolytic cleavage site is indicated. (B) Golgi-localization of the 35S:XYLT: GAUT1 chimera. The construct was expressed transiently in N. benthamiana together with the Golgi marker STtmd-YFP. Scale bar $=50 \mu \mathrm{m}$. (C) Analysis of the rescue of the pollen phenotypes by pGAUT1::XYLT: GAUT1 chimera construct in gaut5 $5^{-/}$gaut6 ${ }^{-/-}$gaut $7^{+-}$mutant (line 897-10). The empty vector in the same mutant was used as a negative control. Distributions of pollen germination phenotypes: "non-germinating" pollen, "bursting" pollen, and pollen with "elongating" tubes (more than 330 pollen of three plants were counted; SEM). (D) Quantification of the length of the pollen tubes ( $\mathrm{n} \geq 120$; SEM). * and ** indicate significant differences as determined by One-way ANOVA ( $p<0.05$ and $p<0.01$, respectively). NS indicates not significantly different.

transformation and the segregation pattern of the GAUT7 and gaut7 alleles was analyzed by PCR in T2 generation. If the chimeric GAUT1 restores the male fertility, the segregation pattern of 3 (WT): $5\left(\right.$ gaut $\left.7^{+/-}\right): 2\left(\right.$ gaut $\left.^{-1-}\right)$ was expected (Supplementary Figure 8). The T2 population derived from the control line, wherein the empty vector was inserted, maintained the same transmission frequencies as observed for the untransformed gaut $^{-/-}$gaut $^{-1-}$ gaut $7^{+-}$triple mutant (Table 1). On the other hand, the T2 populations from two independent lines bearing the chimeric construct (lines 897-10 and 897-14) segregated in skewed ratios (22:24:22 and 20:29:22) (Table 1) and, importantly, we were able to recover rescued homozygous triple gaut5 gaut6 gaut7 mutants. These results confirm that the previous inability to recover homozygous gaut5 gaut6 gaut7 triple mutants is due to a lack of GAUT1synthesized HG due to a lack of retention of GAUT1 in the Golgi.

We further analyzed the control, and the chimera-containing 897-10 T1 line for in vitro pollen germination and pollen tube elongation. In this line the high proportion of defective pollen ("non-germinating") observed in the negative control line (Figure 6C) was complemented to WT-levels. The low proportion of elongating pollen and the increased proportion of bursting pollen, however, remained similar to the negative control level. Partial complementation was also observed for the length of elongating pollen tubes (Figure 6D). These results suggest that the GAUT1 chimera, at least partially, is able to complement the loss of function of the three GAUT anchor proteins.

\section{DISCUSSION}

Homogalacturonan, the most abundant and structurally simplest pectin, has roles in many stages of plant vegetative and reproductive development. While the current understanding of how HG is synthesized in planta by the different members of the GAUT gene family is far from complete, it is becoming increasingly clear that the different members of the gene family produce HG with unique functions (Atmodjo et al., 2013; Amos et al., 2018; Biswal et al., 2018). Here we showed that three different GAUT proteins, GAUT5, GAUT6, and GAUT7, interact with GAUT1, and thereby tether and retain the Nterminally cleaved HG : GalAT to the Golgi apparatus, the location of HG synthesis. This function of the three GAUTs appears to be redundant during the vegetative growth stage, as demonstrated by the WT-like growth of the homozygous single, homozygous double, and even the heterozygous triple mutant plants. However, they are critically required for normal reproduction, specifically in the male gametophyte. The gaut5 $5^{-1}$ gaut $^{-1-}$ gaut $7^{+-}$triple mutant lacking these GAUT1-anchoring proteins was severely defective in pollen germination and tube elongation. A GAUT1 chimera protein capable of self-tethering to the Golgi apparatus was able to partially restore the pollen functions. These observations show that GAUT5, GAUT6, and GAUT7 each tether GAUT1 in the Golgi enabling HG synthesis in the pollen tube.

Mutation of GAUT5, GAUT6, and GAUT7 have differing effects on the deposition of methylesterified HG at the apex of 
pollen tubes, a process critical for native pollen tube growth and male fertility. The binding pattern of the mAb JIM 7, which recognizes highly methylesterified $\mathrm{HG}$, was altered in the growing pollen tubes of all the mutants, even the homozygous gaut5, gaut6, and gaut7 single mutants. The defect was increasingly more severe in the homozygous double and heterozygous triple mutants, resulting in significantly reduced pollen germination and pollen tube growth, and in infertility in the gaut $5^{-1-}$ gaut $^{/-}$gaut $7^{+-}$ mutant leading to the inability to isolate gaut $5^{-1-}$ gaut $^{/-}$gaut $^{-1-}$ homozygous triple mutant plants. The introduction of a GAUT1 chimera protein capable of self-tethering to the Golgi apparatus allowed a successful recovery of the rescued homozygous triple mutant, thus confirming the importance of GAUT5, GAUT6, and GAUT7 function to localize GAUT1 to the Golgi for HG biosynthesis and male gametophyte development.

Growing pollen tubes, as single cells with fast tip-growth facilitated mainly by $\mathrm{HG}$, provide a unique system to start teasing apart the biological functions imparted by GAUT1, GAUT5, GAUT6, GAUT7, their protein:protein interactions and the resulting protein complexes. This task would be much more difficult to carry out using other plant parts that consist of multiple different tissue and cell types. Almost all the Arabidopsis GAUTs are expressed at some level in pollen (Loraine et al., 2013), the most highly expressed being GAUT13 and GAUT14 whose functions in HG synthesis in pollen and pollen tube growth have previously been demonstrated (Wang et al., 2013). It is interesting to note that in pollen, when compared to the transcript levels of GAUT13 and 14, GAUT1 transcript level was reported to be 6 to 9 times lower, and those of GAUT5, GAUT6, and GAUT7 were even lower than GAUT1 (Loraine et al., 2013), yet these three proteins still play significant roles in ensuring a proper plant reproduction process. It would be interesting to investigate the precise roles of GAUT13 and GAUT14 versus the GAUT1-containing HG : GalAT complexes in HG biosynthesis in pollen and pollen tubes.

We envisage that the diversified GAUT protein tethering mechanism can expand the biosynthetic capabilities of GAUT1. It was previously shown that GAUT1 and GAUT7 form the core of an HG : GalAT complex, which transiently interacts with a number of associating proteins including putative methyltransferases in Arabidopsis suspension culture cells (Atmodjo et al., 2011). Furthermore, a recent detailed biochemical analyses of heterologously expressed GAUT1:GAUT7 complex revealed that GAUT1 alone expresses rather poorly compared to when it is coexpressed with GAUT7, and that the HG : GalAT activity of GAUT1 alone is much lower compared to the GAUT1:GAUT7 complex (Amos et al., 2018). Based on these previous findings, we proposed two possibilities for how GAUT1-anchoring by GAUT5, GAUT6, and GAUT7 may have significance beyond Golgi localization. These categories may not be mutually exclusive, but may overlap with each other.

One possibility is that the interaction and/or complex formation with GAUT5, GAUT6, and GAUT7 may impart changes in GAUT1 enzymatic function, with regards to, for example, enzyme kinetics and/or HG product size. Yin et al. (2010b) pointed out that GAUT5 and GAUT7, but not GAUT6, lack a potential nucleophilic threonine residue in the predicted catalytic site (Supplementary Figure 2). It is therefore possible that GAUT5 and GAUT7 play a non-catalytic structural role, while GAUT6 may have a transferase activity. The latter may function as a catalytic subunit in the synthesis of HG backbone, either in association with GAUT1 or independently. Thus, multiple forms of catalytic cores may exist, consisting of GAUT1 alone, GAUT1:GAUT6 complex, or GAUT6 alone, and each of these catalytic core could further associate with a structural subunit (e.g., GAUT5 and GAUT7). Furthermore, the tethering proteins could control the physical location of GAUT1 within the Golgi cisternae. For instance, these proteins appear to share a common motif, Arg-Arg-Trp-x-ArgR/ Lys, in the N-terminal domain localized to the cytosol (Supplementary Figure 2). Varieties of arginine-based motifs have been shown to affect the dynamics of the trafficking of type II membrane proteins between ER and Golgi and possibly within the Golgi cisternae (Dancourt and Barlowe, 2010; Banfield, 2011). It is interesting to note that GAUT7 possesses 12 consecutive Gly residues adjacent to the motif. The function of the Gly residue stretch remains to be elucidated, but it might influence the trafficking of GAUT7 in and around the Golgi apparatus differently from GAUT5 and GAUT6, and thus, affect the precise structure of the synthesized HG. Determination of the precise unique molecular functions of GAUT5, GAUT6, and GAUT7 will require in vitro reconstitution and enzyme kinetics studies of the different GAUT1-containing complexes, as well as analysis of HG produced by the different complexes.

The second possibility is that the different HG biosynthetic GAUT1-containing complexes may interact with distinct sets of associating proteins in the Golgi, during HG trafficking through the secretory pathway, and/or in muro. For example, GAUT5, GAUT6, and GAUT7 may each mediate interaction of the GAUT1 HG : GalAT complexes with different pectin methyltransferases (Atmodjo et al., 2011) and/or pectin acetyltransferases in the Golgi apparatus, resulting in HG products with potentially diverse degrees and/or patterns of esterification. GAUT5, GAUT6, and GAUT7 might also influence interaction of GAUTs with different vesicle trafficking proteins, resulting in transport of different HG products to specific destinations at the cell periphery for deposition into the wall. Such possibilities are in agreement with how the single and double gaut mutants affect the degree and pattern of mAb JIM7 binding in the pollen tip and shaft. Upon incorporation into the wall, the newly deposited, highly methylesterified HG may be recognized as a preferred substrate, based on the specific degree and pattern of esterification, by specific pectin methyl esterases and other pectin modifying enzymes. Indeed, transcriptomics and proteomics studies have shown that at least 14 pectin methylesterases (PMEs), 21 pectin methylesterase inhibitors (PMEIs), and 16 pectin lyases/pectin lyase-like proteins (PLLs) are highly and/or specifically expressed in pollen and pollen tubes (Loraine et al., 2013; Mollet et al., 2013), indicating the importance of HG modification for normal male gametophytic transmission. Determination of the unique molecular functions of GAUT5, GAUT6, and GAUT7 will require identification of the associating protein partners of the different GAUT1-containing 
protein complexes and the HG substrate specificities of the diverse pectin modifying enzymes.

Rapid pollen tube germination and elongation is a hallmark of angiosperms, which dramatically shortened the reproductive cycle and enabled angiosperms to evolve and diversify. As HG plays a central role in pollen tube germination and elongation, the evolution of a multi GAUT-mediated HG synthesis strategy might have provided a competitive advantage for the rate and control of HG synthesis in the pollen tube, thereby supporting rapid pollen tube growth and structural integrity during it race through the pistil to the egg.

In conclusion, we discovered the existence of previously unappreciated complexity in the biosynthesis of one of the most structurally simple cell wall polysaccharides in pollen tubes. The results underscore the complexity and need for rapid biosynthesis and deposition of HG in the highly dynamic male gametophyte in flowering plants. This work paves the way for future studies aimed at deciphering the molecular mechanisms of how the different GAUT anchors and catalytic subunits achieve rapid HG synthesis and support rapid pollen tube growth.

\section{DATA AVAILABILITY STATEMENT}

All datasets presented in this study are included in the article/ supplementary material.

\section{AUTHOR CONTRIBUTIONS}

YS, CL, AS, and JM conceived the experiment, and together with MA, AB, SE, DM, IM, and RR carried it out. YS, CL, AS, JM, MA,

\section{REFERENCES}

Alexander, M. P. (1969). Differential Staining of Aborted and Nonaborted Pollen. Stain Technol. 44, 117-122. doi: 10.3109/10520296909063335

Amos, R. A., Pattathil, S., Yang, J.-Y., Atmodjo, M. A., Urbanowicz, B. R., Moremen, K. W., et al. (2018). A two-phase model for the non-processive biosynthesis of homogalacturonan polysaccharides by the GAUT1:GAUT7 complex. J. Biol. Chem. 293, 19047-19063. doi: 10.1074/jbc.RA118.004463

Anderson, C. T. (2016). We be jammin': an update on pectin biosynthesis, trafficking and dynamics. J. Exp. Bot. 67, 495-502. doi: 10.1093/jxb/erv501

Atmodjo, M. A., Sakuragi, Y., Zhu, X., Burrell, A. J., Mohanty, S. S., Atwood, J. A. 3., et al. (2011). Galacturonosyltransferase (GAUT)1 and GAUT7 are the core of a plant cell wall pectin biosynthetic homogalacturonan:galacturonosyltransferase complex. Proc. Natl. Acad. Sci. 108, 20225-20230. doi: 10.1073/pnas.1112816108

Atmodjo, M. A., Hao, Z., and Mohnen, D. (2013). Evolving views of pectin biosynthesis. Annu. Rev. Plant Biol. 64, 747-779. doi: 10.1146/annurevarplant-042811-105534

Banfield, D. K. (2011). Mechanisms of protein retention in the Golgi. Cold Spring Harb. Perspect. Biol. 3:a005264. doi: 10.1101/cshperspect.a005264

Biswal, A. K., Atmodjo, M. A., Li, M., Baxter, H. L., Yoo, C. G., Pu, Y., et al. (2018). Sugar release and growth of biofuel crops are improved by downregulation of pectin biosynthesis. Nat. Biotechnol. 36, 249-257. doi: 10.1038/nbt.4067

Caffall, K. H., and Mohnen, D. (2009). The structure, function, and biosynthesis of plant cell wall pectic polysaccharides. Carbohydr. Res. 344, 1879-1900. doi: 10.1016/j.carres.2009.05.021

Caffall, K. H., Pattathil, S., Phillips, S. E., Hahn, M. G., and Mohnen, D. (2009). Arabidopsis thaliana T-DNA mutants implicate GAUT genes in the
$\mathrm{AB}$, and $\mathrm{RR}$ designed and carried out the data analysis. YS, CL, $\mathrm{MA}, \mathrm{AB}, \mathrm{DM}$, and JM wrote and edited the manuscript. All authors contributed to the article and approved the submitted version.

\section{FUNDING}

We thank the Danish Council for Independent Research (project no. 272-07-0152 and 8022-00222B), the Villum Fonden (projects no. 13363 and 17489), and the Center for Bioenergy Innovation, a U.S. Department of Energy Bioenergy Research Center supported by the Office of Biological and Environmental Research in the DOE Office of Science, for funding.

\section{ACKNOWLEDGMENTS}

We thank the WallNet consortium and Dr. Henrik V. Scheller (Joint Bienergy Institute, USA) for kindly providing GAUT and GATL GATEWAY entry vectors. In addition, we would like to thank Robert D. Hoffmann (University of Copenhagen, DK) for discussions. All confocal microscopy was carried out at the Center for Advanced Bioimaging (University of Copenhagen, DK).

\section{SUPPLEMENTARY MATERIAL}

The Supplementary Material for this article can be found online at: https://www.frontiersin.org/articles/10.3389/fpls.2020.585774/ full\#supplementary-material

biosynthesis of pectin and xylan in cell walls and seed testa. Mol. Plant 2, 1000-1014. doi: $10.1093 / \mathrm{mp} / \mathrm{ssp} 062$

Cantarel, B. L., Coutinho, P. M., Rancurel, C., Bernard, T., Lombard, V., and Henrissat, B. (2009). The Carbohydrate-Active EnZymes database (CAZy): An expert resource for glycogenomics. Nucleic Acids Res. 37, 233-238. doi: 10.1093/nar/gkn663

Chebli, Y., Kaneda, M., Zerzour, R., and Geitmann, A. (2012). The cell wall of the Arabidopsis pollen tube-spatial distribution, recycling, and network formation of polysaccharides. Plant Physiol. 160, 1940-1955. doi: 10.1104/pp.112.199729

Dancourt, J., and Barlowe, C. (2010). Protein sorting receptors in the early secretory pathway. Annu. Rev. Biochem. 79, 777-802. doi: 10.1146/annurevbiochem-061608-091319

Dardelle, F., Lehner, A., Ramdani, Y., Bardor, M., Lerouge, P., Driouich, A., et al. (2010). Biochemical and immunocytological characterizations of Arabidopsis pollen tube cell wall. Plant Physiol. 153, 1563-1576. doi: 10.1104/ pp. 110.158881

Ferguson, C., Teeri, T. T., Siika-aho, M., Read, S. M., and Bacic, A. (1998). Location of cellulose and callose in pollen tubes and grains of Nicotiana tabacum. Planta 206, 452-460. doi: 10.1007/s004250050421

Goubet, F., and Mohnen, D. (1999). Solubilization and partial characterization of homogalacturonan-methyltransferase from microsomal membranes of suspension-cultured tobacco cells. Plant Physiol. 121, 281-290. doi: 10.1104/ pp.121.1.281

Grobei, M. A., Qeli, E., Brunner, E., Rehrauer, H., Zhang, R., Roschitzki, B., et al. (2009). Deterministic protein inference for shotgun proteomics data provides new insights into Arabidopsis pollen development and function. Genome Res. 19, 1786-1800. doi: 10.1101/gr.089060.108 
Jungmann, J., and Munro, S. (1998). Multi-protein complexes in the cis Golgi of Saccharomyces cerevisiae with alpha-1,6-mannosyltransferase activity. EMBO J. 17, 423-434. doi: 10.1093/emboj/17.2.423

Li, X. (2011). Pollen Fertility/viability Assay Using FDA Staining. Bio-101, e75. doi: 10.21769/BioProtoc.75

Loraine, A. E., McCormick, S., Estrada, A., Patel, K., and Qin, P. (2013). RNA-Seq of Arabidopsis Pollen Uncovers Novel Transcription and Alternative Splicing. Plant Physiol. 162, 1092-1109. doi: 10.1104/pp.112.211441

Lund, C. H., Bromley, J. R., Stenbæk, A., Rasmussen, R. E., Scheller, H. V., and Sakuragi, Y. (2015). A reversible Renilla luciferase protein complementation assay for rapid identification of protein-protein interactions reveals the existence of an interaction network involved in xyloglucan biosynthesis in the plant Golgi apparatus. J. Exp. Bot. 66, 85-97. doi: 10.1093/jxb/eru401

Mollet, J. C., Leroux, C., Dardelle, F., and Lehner, A. (2013). Cell Wall Composition, Biosynthesis and Remodeling during Pollen Tube Growth. Plants (Basel) 2, 107-47. doi: 10.3390/plants2010107

Mravec, J., Kračun, S. K., Rydahl, M. G., Westereng, B., Miart, F., Clausen, M. H., et al. (2014). Tracking developmentally regulated post-synthetic processing of homogalacturonan and chitin using reciprocal oligosaccharide probes. Development 141, 4841-4850. doi: 10.1242/dev.113365

Munro, S. (1995). An investigation of the role of transmembrane domains in Golgi protein retention. EMBO J. 14, 4695-4704. doi: 10.1002/j.14602075.1995.tb00151.x

Obrdlik, P., El-Bakkoury, M., Hamacher, T., Cappellaro, C., Vilarino, C., Fleischer, C., et al. (2004). K+ channel interactions detected by a genetic system optimized for systematic studies of membrane protein interactions. Proc. Natl. Acad. Sci. U. S. A. 101, 12242-12247. doi: 10.1073/pnas.0404467101

Pauly, M., and Ramírez, V. (2018). New Insights Into Wall Polysaccharide OAcetylation. Front. Plant Sci. 9:1210. doi: 10.3389/fpls.2018.01210

Perrone, P., Hewage, C. M., Thomson, A. R., Bailey, K., Sadler, I. H., and Fry, S. C. (2002). Patterns of methyl and O-acetyl esterification in spinach pectins: new complexity. Phytochemistry 60, 67-77. doi: 10.1016/S0031-9422(02)00039-0

Qin, Y., Leydon, A. R., Manziello, A., Pandey, R., Mount, D., Denic, S., et al. (2009). Penetration of the Stigma and Style Elicits a Novel Transcriptome in Pollen Tubes, Pointing to Genes Critical for Growth in a Pistil. PLoS Genet. 5, e1000621. doi: 10.1371/journal.pgen.1000621

Rédei, G. P. (1965). Non-mendelian megagametogenesis in Arabidopsis. Genetics 51, 857-872.

Robertson, W. R., Clark, K., Young, J. C., and Sussman, M. R. (2004). An Arabidopsis thaliana plasma membrane proton pump is essential for pollen development. Genetics 168, 1677-1687. doi: 10.1534/genetics.104.032326

Rodriguez-Enriquez, M. J., Mehdi, S., Dickinson, H. G., and Grant-Downton, R. T. (2013). A novel method for efficient in vitro germination and tube growth of Arabidopsis thaliana pollen. New Phytol. 197, 668-679. doi: 10.1111/nph.12037

Saint-Jore-Dupas, C., Gomord, V., and Paris, N. (2004). Protein localization in the plant Golgi apparatus and the trans-Golgi network. Cell. Mol. Life Sci. 61, 159171. doi: 10.1007/s00018-003-3354-7

Sakuragi, Y., Nørholm, M. H., and Scheller, H. H. V. (2011a). In The Plant Cell Wall. Ed. Z. A. Popper (Totowa, New Jersey, USA: Humana Press). 153-168

Sakuragi, Y., Nørholm, M. H., and Scheller, H. H. V. (2011b). Visual mapping of cell wall biosynthesis. Methods Mol. Biol. 715, 153-167. doi: 10.1007/978-161779-008-9_11
Schoberer, J., Liebminger, E., Botchway, S. W., Strasser, R., and Hawes, C. (2013) Time-resolved fluorescence imaging reveals differential interactions of $\mathrm{N}$ glycan processing enzymes across the Golgi stack in planta. Plant Physiol. 161, 1737-1754. doi: 10.1104/pp.112.210757

Smyth, D. R., Bowman, J. L., and Meyerowitz, E. M. (1990). Early flower development in Arabidopsis. Plant Cell 2, 755-767. doi: 10.1105/tpc.2.8.755

Stagljar, I., Korostensky, C., Johnsson, N., and te Heesen, S. (1998). A genetic system based on split-ubiquitin for the analysis of interactions between membrane proteins in vivo. Proc. Natl. Acad. Sci. U. S. A. 95, 5187-5192. doi: $10.1073 /$ pnas.95.9.5187

Sterling, J. D., Quigley, H. F., Orellana, A., and Mohnen, D. (2001). The catalytic site of the pectin biosynthetic enzyme alpha-1,4-galacturonosyltransferase is located in the lumen of the Golgi. Plant Physiol. 127, 360-371. doi: 10.1104/ pp.127.1.360

Sterling, J. D., Atmodjo, M. A., Inwood, S. E., Kumar Kolli, V. S., Quigley, H. F., Hahn, M. G., et al. (2006). Functional identification of an Arabidopsis pectin biosynthetic homogalacturonan galacturonosyltransferase. Proc. Natl. Acad. Sci. U. S. A. 103, 5236-5241. doi: 10.1073/pnas.0600120103

Stranne, M., Ren, Y., Fimognari, L., Birdseye, D., Yan, J., Bardor, M., et al. (2018). TBL10 is required for O-acetylation of pectic rhamnogalacturonan-I in Arabidopsis thaliana. Plant J. 96, 772-785. doi: 10.1111/tpj.14067

Taylor, L. P., and Hepler, P. K. (1997). Pollen germination and tube growth. Annu. Rev. Plant Physiol. Plant Mol. Biol. 48, 461-491. doi: 10.1146/annurev.arplant. 48.1.461

Wang, Y., Zhang, W. Z., Song, L. F., Zou, J. J., Su, Z., and Wu, W. H. (2008). Transcriptome Analyses Show Changes in Gene Expression to Accompany Pollen Germination and Tube Growth in Arabidopsis. Plant Physiol. 148, 1201-1211. doi: 10.1104/pp.108.126375

Wang, L., Wang, W., Wang, Y. Q., Liu, Y. Y., Wang, J. X., Zhang, X. Q., et al. (2013). Arabidopsis galacturonosyltransferase (GAUT) 13 and GAUT14 have redundant functions in pollen tube growth. Mol. Plant 6, 1131-1148. doi: $10.1093 / \mathrm{mp} / \mathrm{sst} 084$

Yin, Y., Chen, H., Hahn, M. G., Mohnen, D., and Xu, Y. (2010a). Evolution and function of the plant cell wall synthesis-related glycosyltransferase family 8 . Plant Physiol. 153, 1729-1746. doi: 10.1104/pp.110.154229

Yin, Y., Mohnen, D., Gelineo-Albersheim, I., Xu, Y., and Hahn, M. G. (2010b). "Glycosyltransferases of Family 8 (GT8)," in Plant Cell Wall Polysaccharides: Biosynthesis and Bioengineering, Annual Plant Review series (Oxford, UK: Blackwell).

Conflict of Interest: The authors declare that the research was conducted in the absence of any commercial or financial relationships that could be construed as a potential conflict of interest.

Copyright (c) 2020 Lund, Stenboe, Atmodjo, Rasmussen, Moller, Erstad, Biswal, Mohnen, Mravec and Sakuragi. This is an open-access article distributed under the terms of the Creative Commons Attribution License (CC BY). The use, distribution or reproduction in other forums is permitted, provided the original author(s) and the copyright owner(s) are credited and that the original publication in this journal is cited, in accordance with accepted academic practice. No use, distribution or reproduction is permitted which does not comply with these terms. 